International Journal of Engineering \& Technology, $7(4.38)(2018) 1392-1394$
International Journal of Engineering \& Technology
Website: $w$ ww.sciencepubco.com/index.php/IJET
Research paper

\title{
Brain Image Segmentation with Gradient Information
}

\author{
Sri Purwani1, Julita Nahar1 and Carole Twining2 \\ 1Department of Mathematics, Universitas Padjadjaran, Bandung, Indonesia \\ 2Imaging Sciences, School of Medicine, Faculty of Medical and Human Sciences, The University of Manchester, United Kingdom \\ *Corresponding author E-mail:sri.purwani@unpad.ac.id
}

\begin{abstract}
Segmentation is the process of extracting structures within the images. The purpose is to simplify the representation of the image into something meaningful and easier to analyse. A magnetic resonance (MR) brain image can be represented as three main tissues, e.g. cerebrospinal fluid (CSF), grey matter and white matter. Although various segmentation methods have been developed, such images are generally segmented by modelling the intensity histogram by using a Gaussian Mixture Model (GMM). However, the standard use of 1D histogram sometimes fails to find the mean for Gaussians. We hence solved this by including gradient information in the 2D intensity and intensity gradient histogram. We applied our methods on real data of 2D MR brain images. We then compared the methods with the previous published method of Petrovic et al. on their dataset, as well as on our larger datasets extracted from the same database of 3D MR brain mages, where the ground-truth annotations are available. This shows that our method performs better than the previous method.
\end{abstract}

Keywords: Image segmentation, tissue fraction image, Gaussian Mixture Model with partial voluming

\section{Introduction}

Segmentation is the process of identifying structures in the images. The purpose is to simplify the representation of the image into something meaningful and easier to analyze [Shapiro and Stockman, 2001]. One simple segmentation method uses a closed contour, which divides the image region into inside and outside [Chan and Vese, 2001]. The initial standard ACM on edges/lines was introduced by Kass et al. [Kass et al., 1988].

Unal and Slabaugh used a region-based, pairwise Active Contour approach [Unal G and Slabaugh, 2005]. This allows segmentation of one image to help guide the segmentation of another, and proceeds without an initial segmentation, so that the segmenting contour evolves in both images. However, the registration and resultant image correspondence is only defined within a narrow band surrounding each segmenting contour/surface.

According to Ashburner and Friston [Ashburner and Friston, 2005], segmentation of brain images generally uses either a tissue classification approach or registration to a template. They combine both processes, and use an initial tissue probability atlas in order to perform registration, intensity correction, and segmentation of brain images. However, this was only shown on BrainWeb simulated images and used 'anecdotal validation.

Furthermore, in [Pohl et al., 2006] they combined atlas registration with segmentation of MR brain images to produce segmentations of brain tissues, as well as their substructures. The method uses labelmaps information and known intensity histograms to create an artificial intensity reference to better fit the training image. However, the atlas needs manual and semi-automatic segmentations of up to 80 different brains, and the method was only applied to 22 brain images.

This survey of segmentation methods shows that the earlier edgebased methods of segmentation [Kass et al., 1988], tended to be replaced by region-based methods, and most methods are unsu- pervised. For unsupervised segmentation, many methods require registration to an atlas [Unal G and Slabaugh, 2005: Ashburner and Friston, 2005: Pohl et al., 2006: Chegini and Ghassemian, 2011: Ahmadvand and Kabiri, 2014]. However, if prior knowledge in terms of an atlas is not available, then a method that does not require training data for segmentation is necessary If we consider the classes of segmentation algorithm described in [Pham et al., 2000], then in terms of automated methods that do not require training, the only candidate is clustering-type methods. For dense data, clustering becomes in effect a Gaussian mixture model, hence we come to the Gaussian mixture model methods as used by Petrovic et al. [Petroviф et al., 2007]. These methods are more sophisticated than the simple Gaussian mixture models, since they also consider partial-voluming effects. We used our methods to segment MR brain images discussed in the following section.

\section{Segmentation Methods}

Given a slice of brain image, we model the intensity histogram as a set of Gaussians (plus possible partial-volume effects). We assume that the brain image has 3 main tissues (CSF, grey matter and white matter), and mixtures between two pure tissues. In [Petrovic et al., 2007], the 1D GMM fitting procedure used a Greedy algorithm to find three main peaks. These peaks give the means for the three Gaussians. However, when the GMM and fitting methods were applied to the larger dataset, the model failed to fit to many images (see e.g., Figure 1(a), where the model (black line) failed to locate the pure grey matter Gaussian). 


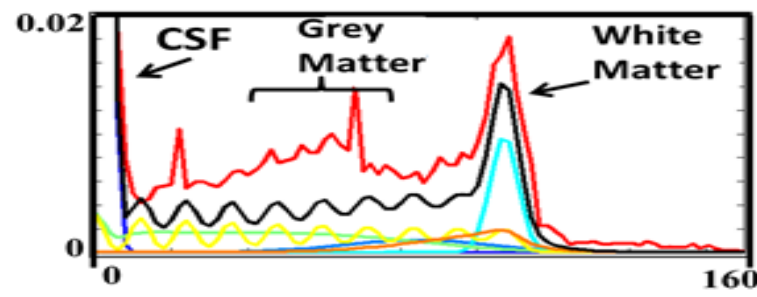

(A) 1D histogram method

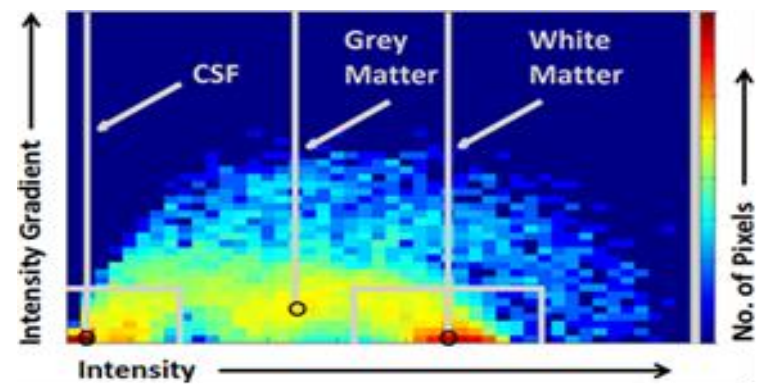

(B) 2D histogram method.

Figure 1. The standard intensity 1D histogram (Left) and the new intensity-gradient 2D histogram (Right). (a) The red line is the image histogram, and the black line is the failed GMM fit, which consists of a sum over 3 pure tissue Gaussians, plus 3 mixtures (coloured lines); (b) The yellow/red regions at the feet of the yellow arcs give the new estimates of the puretissue means (shown by the vertical grey lines and the black circles). The boxes are the excluded zones.

Therefore, we need a more robust GMM fitting procedure than the simple $1 \mathrm{D}$ one. We obtain this by using the $2 \mathrm{D}$ intensity valueand-gradient histogram (see Figure 1(b)). The gradient, which describes intensity changes within images, is highest at the boundary between tissues. Hence, it can separate partial-volume boundary pixels from the pure-tissue pixels (see [Thacker et al., 2015: Williamson et al., 2002] for further details). As can be seen in Figure 1(b), the pure tissue peaks are located at the feet of the yellow arcs that correspond to partial-volume mixtures of tissues (for similar arcs, see Figure 2 in [Williamson et al., 2002] and Figure 5 in [Thacker et al., 2015]).

The intensity values for these peaks are used as the mean positions for the three Gaussians which are kept fixed during the optimization of the fit of the 1D histogram. We optimize all the other parameters such as variance and weights by using Sum of Squared Differences. It was found that this procedure works on all images in the larger dataset without fail, and gives a good fit for all the 1D histograms. Having a robust GMM fitting procedure, we then use the model to create an intensity-to-fraction-values mapping. This gives fraction images discussed in the following section.

\section{Fraction Images}

The intensity distribution of each tissue is then used to estimate the tissue fraction in each pixel. Between the two pure-tissue means, this computes a fraction value $f$ thus:

$\mu_{i}<g<\mu_{j}: g=f \mu_{i}+(1-f) \mu_{j}$

$\Rightarrow f=1-\frac{g-\mu_{i}}{\mu_{j}-\mu_{i}}, \quad f_{i}=f, f_{j}=1-f$

Alternatively, as the value of $f$ at a particular intensity $g$ can be chosen to maximize the mixture-Gaussian probability $p(g \| f)$ over the whole range of $f$ [Petrovic et al., 2007]: $f=\arg \max _{f} p(g \mid f)$

With three tissues, and with the three means for the pure tissue Gaussians, we first compute the following functions:

$f_{i, i+1}(g)=\left\{\begin{array}{lc}1, & g \leq \mu_{i} \\ 1-\frac{g-\mu_{i}}{\mu_{j}-\mu_{i}}, & \mu_{i}<g<\mu_{i+1}, \quad i=1,2 \\ 0, & g \geq \mu_{i+1}\end{array}\right.$

where $g$ is the intensity, and $\left\{\mu_{j} j=1,2,3\right\}$ are the three ordered pure-tissue mean intensities for CSF, grey matter and white matter. The three tissue fractions are then given by:

$$
\begin{array}{cc}
\text { CSF : } & f_{1}(g)=f_{1,2}(g), \\
\text { grey matter: } & f_{2}(g)=f_{2,3}(g)-f_{1,2}(g) \\
\text { whitematter : } & f_{3}(g)=1-f_{2,3}(g), \\
f_{1}(g)+f_{2}(g)+f_{3}(g) \equiv 1 \quad \forall g
\end{array}
$$

This gives a continuous piecewise-linear mapping which can preserve the pure tissue pixels (see e.g. a resultant fraction image from the continuous piecewise-linear mapping, bottom row in Figure 2).
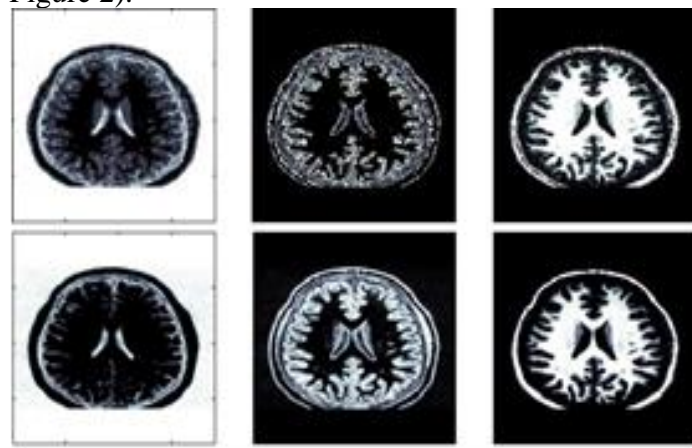

Figure 2. Tissue fractions of a single image given by Petrovic's maximum responsibility method (1) (Top), and the linear mapping (3) (Bottom), for (Left to Right) CSF, grey matter and white matter.

Evaluation Segmentation Methods

We have ground-truth manual annotations (grouped label images) available with our datasets. We also have tissue fraction images for each image across the sets from the segmentation. This allows us to perform evaluation of the segmentation methods by using Tanimoto overlap values [Crum et al., 2006].This measures the amount of overlap between grouped-label images (see e.g. Figure 3 ) and tissue fraction images (see e.g. Figure 2), with the calculation for image and tissue is as follows,

Where and run over all pixels in the image

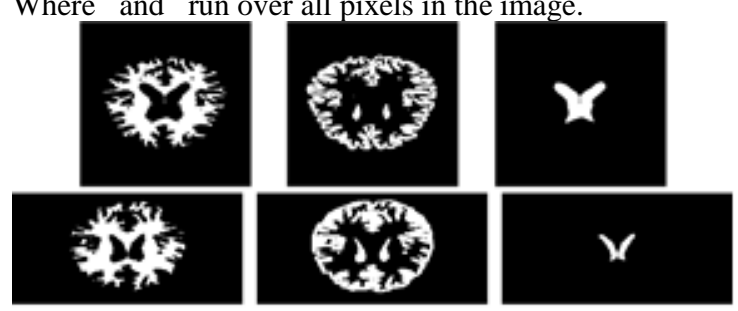

Figure 3. Left to right three main ground-truth labels; white matter, grey matter and CSF from the 37-dataset (Top) and 52-dataset (Bottom), respectively. Those are for different slices of MR brain images.

The fraction images from the 2D histogram method are then compared to those from the 1D histogram method [Petroviф et al., 
2007], by using Tanimoto overlap values with ground-truth [Crum et al., 2006] of each image on the 37-dataset.

Table 1. Means and standard deviations of Tanimoto value distributions by using the $1 \mathrm{D}$ and $2 \mathrm{D}$ methods on the 37 -dataset.

\begin{tabular}{|l|l|l|l|l|}
\hline \multirow{2}{*}{ Tissues } & 1D & \multicolumn{2}{l|}{ 2D } \\
\cline { 2 - 5 } & Mean & Std. & Mean & Std. \\
\hline White matter & 0.66 & 0.08 & 0.68 & 0.08 \\
\hline Grey matter & 0.38 & 0.13 & 0.48 & 0.06 \\
\hline CSF & 0.83 & 0.04 & 0.84 & 0.03 \\
\hline
\end{tabular}

This table shows the 2D method has higher mean values than the 1D method for all tissue fractions, as well as smaller standard deviation for grey matter and CSF (see e.g. a resultant fraction image from 2D method, bottom row in Figure 2). The same results are also observed on the other datasets,

\section{Conclusions and Future Work}

Including gradient information in the 2D histogram, shows two strong arches, with three feet which correspond to the three means of the pure tissue Gaussians (see Figure 1(b), and see also in [Thacker et al., 2015: Williamson et al., 2002]). This produces a robust model fit which can fit histograms of all the images in the set including the ones where Petrovic's method fails. The model and means are then used to create mappings for segmentation.

Table 1 shows the 2D method has higher mean values than the $1 \mathrm{D}$ method for all tissue fractions, as well as smaller standard deviation for grey matter and CSF. This shows 2D method has better fraction images than the 1D method. This result also applies for the other larger datasets.

With better segmentation of each and every image, we can then use tissue fraction images as parts of registration stage. This supports our aim of integrating segmentation and registration to improve the results.

\section{Acknowledgements}

Ms Purwani would like to thank the Directorate General of Higher Education of Indonesia (DIKTI) for providing funding and completing her $\mathrm{PhD}$. We also thank the University of Padjadjaran for financial support during the completion of the paper.

\section{References}

[1] Ahmadvand, A., and Kabiri, P., 2014. Multispectral MRI image segmentation using markov random field model. Signal, Image and Video Processing, pages 1-8, 10.1007/s11760-014-0734-4.

[2] Ashburner, J., and Friston, K. J., 2005. Unified segmentation. NeuroImage, 26(3):839-851.

[3] Chan, T. F., and Vese, L. A., 2001. Active contours without edges. IEEE Transactions on Image Processing, 10(2):266-277.

[4] Chegini, M., and Ghassemian, H., 2011. Spatial spectral gaussian mixture model approach for automatic segmentation of multispectral MR brain images. In Electrical Engineering (ICEE), 2011 19th Iranian Conference, pages 1-6.

[5] Crum, W., Camara, O., and Hill, D., 2006. Generalised overlap measures for evaluation and validation in medical image analysis. IEEE Transactions on Medical Imaging, 25(11):1451-1461.

[6] Kass, M., Witkin, A., and Terzopoulos, D., 1988. Snakes: Active contour models. International Journal of Computer Vision, 1:321 331.

[7] Petroviф, V., Cootes, T. F., Twining, C. J., and Taylor, C. J., 2007 Simultaneous registration, segmentation, and modelling of structure in groups of medical images. In 4th IEEE International Symposium on Biomedical Imaging (ISBI): From Nano to Macro, pages 1-4

[8] Pham, D. L., Xu, C., and Prince, J. L., 2000. Current methods in medical image segmentation. Annual review of biomedical engineering, 2:315-338.

[9] Pohl, K. M., Fisher, J., Grimson, W. E. L., Kikinis, R., and Wells W. M., 2006. A Bayesian model for joint segmentation and registration. NeuroImage, 31(1):228-239.
[10] Shapiro, L. G., and Stockman, G. C., 2001. Computer Vision. New Jersey. Prentice-Hall.

[11] Thacker, N. A., Bromiley, P. A., and Williamson, D. C., 2015. Multi-dimensional medical image segmentation with partial volume and gradient modelling. TINA memos, available online: http://www.tina-vision.net/docs/memos/2004-009.pdf Accessed January 2015.

[12] Unal, G., and Slabaugh, G., 2005. Coupled PDEs for non-rigid registration and segmentation. In Proc. of the IEEE computer society conference on computer vision and pattern recognition (CVPR), volume 1, pages 168-175.

[13] Williamson, D. C., Thacker, N. A., Williams, S. R., and Pokric, M., 2002. Partial volume tissue segmentation using grey-level gradient. In Proceedings of Medical Image Understanding and Analysis (MIUA), volume 2, pages 17-20 BMVA. 